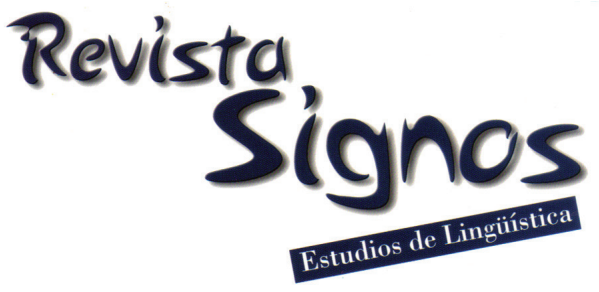

\title{
El desarrollo narrativo a través de la adolescencia: Estructura global de contenido y referencia personal
}

\author{
Narrative development across adolescence: Global content structure \\ and personal referente
}

\author{
Soledad Aravena \\ solaravena@gmail.com \\ Universidad Alberto Hurtado \\ Chile
}

Recibido: 7-IX-2010 / Aceptado: 2I-IV-20II

Resumen: Esta investigación se propone describir las formas lingüísticas de la referencia personal y las funciones narrativas (orientación, episodios, etc.) que se despliegan después de la infancia. Se analizaron 160 relatos orales y escritos de 80 nativos del español peninsular (40 hombres y 40 mujeres), pertenecientes a cuatro niveles de edad/ escolarización: 9, 12 y 16 años más un grupo de adultos universitarios (25-30 años). Cada participante produjo un texto oral y otro escrito sobre un tema elicitado por un video sobre los conflictos interpersonales en una escuela. Los resultados muestran que el efecto de la edad es significativo en la configuración estructural de las narraciones, particularmente respecto de los componentes eventivos y evaluativos. Se observa también que los niños de 9 y 12 años introducen mucha información que elaboran poco y utilizan los episodios y la complicación tanto para introducir como para mantener los referentes. En cambio, los grupos mayores introducen pocos referentes que mantienen eficientemente a lo largo del discurso y destinan distintos componentes estructurales para introducir la referencia y para mantenerla. Se concluye que después de los 10 años hay cambios significativos en la producción del discurso, vinculados con distintos modos de procesamiento, que corroboran la idea del desarrollo lingǘstico como un proceso prolongado que se extiende hasta la adultez.

Palabras Clave: Desarrollo tardío del lenguaje, narración, referencia personal. 


\begin{abstract}
The study aims to describe the linguistic forms of personal reference and the narrative functions (orientation, episodes, etc.) developed after childhood. 160 oral and written narratives were analyzed, from 80 native peninsular Spanish speakers ( 40 men and 40 women) belonging to four age/schooling groups: 9,12 , and 16 years old, plus a graduate adult group (25-30 years old). All participants produced a written and an oral text on a subject elicited by a video on interpersonal conflict in a school. The results show that the effect of age is significant in the structural configuration of the narratives, particularly in the eventive and evaluative components. It is also observed that children in the 9 and 12 year olds groups introduce plenty of information that they do not elaborate effectively. In addition, they use episodes and complications both to introduce and maintain referents. In contrast, older subjects tend to introduce a few referents, which are efficiently maintained throughout the discourse. Moreover, they use different structural components to introduce and to maintain referents. Findings suggest that after 10 years old, there are significant changes in discourse production, linked to different processing modes, supporting the idea of linguistic development as a protracted process that extends into adulthood.
\end{abstract}

Key Words: Later language development, narrative, personal reference.

\section{INTRODUCCIÓN}

En los últimos años, la investigación acerca de los desarrollos tardíos del lenguaje ha confirmado que el esquema narrativo es un logro temprano. Sin embargo, una vez alcanzado su dominio básico -en torno a los 9 años- las narraciones siguen experimentando cambios durante los años de la adolescencia (Berman \& Nir-Sagiv, 2007; Tolchinsky, 2007). Esta tendencia se ha evidenciado principalmente en relación con la función de los fenómenos léxicos y gramaticales en el texto, pero también, en menor medida, respecto de la estructura global del contenido (Berman, 2008). No obstante, el modo en que evoluciona el dominio de la estructura narrativa en relación con determinadas formas y funciones propias del género en edades posteriores a los 9 años ha sido poco estudiado.

En este contexto, se ha propuesto este trabajo, cuyo objetivo general es: describir evolutivamente la construcción del discurso narrativo en cuanto a las formas lingüísticas de la referencia personal y las funciones narrativas que se despliegan después de la infancia. Los objetivos específicos derivados son:

I. Determinar el modo en que los componentes estructurales de la narración se distribuyen en los textos orales y escritos de cuatro grupos de edad/ escolarización.

2. Determinar la proporción y las formas lingüísticas con que los sujetos introducen y mantienen los referentes de persona en sus textos orales y escritos. 3. Identificar las funciones narrativas que despliegan los sujetos mediante la introducción y la mantención de los referentes de persona en sus textos orales y escritos.

El estudio se enmarca, por lo tanto, en el campo teórico y metodológico de la Psicolingüística y se interesa por los procesos de desarrollo y por la correspondencia de las formas lingüísticas con sus usos y sus funciones comunicativas (Slobin, 1990, 1996). El interés de la investigación radica, por un lado, en el intento por analizar las formas lingüísticas de la referencia personal en relación con las funciones narrativas que dichas formas contribuyen a desplegar. Por otro lado, en el hecho de que se analicen las dos modalidades del discurso en una lengua $y$ en edades poco estudiadas.

En cuanto a la edad de los sujetos, diversos estudios sobre el desarrollo del lenguaje infantil (Berman \& Slobin, 1994; Hickmann, 1995, 2003) han postulado distintos ritmos evolutivos para las formas y las funciones. Es decir, aunque las formas lingüísticas emerjan tempranamente, la habilidad para movilizar múltiples opciones retóricas e integrarlas apropiadamente en las funciones discursivas se adquiere tardíamente (después de los 8 o 10 años) y sigue una larga trayectoria evolutiva (Berman, 1997). Ejemplo de ello son los dispositivos para referir a las personas, objeto del presente estudio. Algunas de estas formas (como sintagmas nominales definidos e indefinidos, pronombres, etc.) se adquieren muy tempranamente. Sin embargo, la habilidad para utilizarlos con funciones intradiscursivas (y no deícticas) específicas se afianza muy tarde, hacia los 10 años (Karmiloff-Smith, 1985; Hickmann, 1995). La edad de 9 años -que marca el límite inferior de edad para los participantes de esta investigaciónresulta, entonces, un hito importante para comenzar a examinar la aparición de las funciones aludidas.

También durante la adolescencia se afianzan muchos usos de la modalidad escrita del discurso, así como la habilidad para organizar el texto atendiendo a sus múltiples requisitos estructurales, semánticos 
y pragmáticos, además de formales y tipográficos. Se presume que construir un texto escrito impone demandas cognitivas a la memoria, funciones ejecutivas y de procesamiento descendente que no están listas antes de la adolescencia (Ravid \& Tolchinsky, 2002; Berman \& Ravid, 2009). Todo lo anterior se examina en la investigación debido a las edades consideradas en la muestra.

\section{Marco teórico}

\section{I.I. Desarrollo del lenguaje más allá de la primera infancia}

La presente investigación se inscribe en el campo de estudio del 'desarrollo tardío del lenguaje' (de ahora en adelante DTL). Su supuesto de base es la evidencia de que los niños de 6 años (y también los de $9 \circ$ 12), aunque ya utilizan correctamente la mayoría de estructuras gramaticales de su lengua, presentan un dominio discursivo menos experto que el de los adultos (Nippold, 1998; Tomasello, 2005; Berman \& Ravid, 2009). Las diferencias no se deben exclusivamente, como podría pensarse, a la poca experiencia vital y lingüística y por ende a un léxico más limitado o a una sintaxis menos compleja. Se trata fundamentalmente de las transformaciones que se producen a través del tiempo en la relación entre formas lingüísticas y funciones discursivas y su uso flexible en distintos contextos comunicativos. El estudio del DTL se interesa, entonces, por los cambios lingüísticos y funcionales observables en el discurso de los niños una vez concluida la fase del desarrollo lingüístico temprano, hacia los $5 \circ 6$ años. En otras palabras, se interesa por los cambios ocurridos entre la 'emergencia' del lenguaje y el 'dominio competente' del mismo (Berman, 2004).

El foco en el nivel discursivo y en las funciones de las formas lingüísticas sitúa al campo del DTL entre las aproximaciones de corte funcional al desarrollo lingüístico (Tomasello, 1998). Según estas, la adquisición es un proceso en que se aprenden los dispositivos lingüísticos como herramientas para alcanzar metas comunicativas. Es decir, las formas lingüísticas se adquieren porque sirven a objetivos cruciales en la comunicación. Así, el cambio evolutivo está tanto o más vinculado con las propiedades semánticas y pragmáticas de las unidades lingüísticas como con sus rasgos estructurales (Hickmann, 2003).

\section{I.2. Desarrollo lingüístico y discurso narrativo}

La narración corresponde a un tipo de 'discurso extendido', es decir, conectado y descontextualizado. Ello implica que no puede construirse sobre la base de las pistas extralingüísticas dadas por el contexto o los conocimientos compartidos con el interlocutor durante una conversación. La dificultad en la producción del discurso extendido no radica, sin embargo, en la necesidad de ser totalmente explícitos y exhaustivos. Más bien, radica en el hecho de que el hablante debe evaluar y decidir de modo preciso cuán explícito y exhaustivo debe ser (Ninio \& Snow, 1996). Además, se trata de discursos que deben utilizar el mensaje como su propio contexto y por lo tanto requieren un sofisticado grado de estructura, coherencia y gestión de la información (Hickmann, 2003).

Así, la narración requiere construir una historia con una estructura temática global y cierta conectividad semántica y lingüística entre las oraciones. Esto supone guiar al oyente desde el planteamiento inicial a través del conflicto y hasta el desenlace ligando las cadenas de oraciones mediante recursos lingüísticos (como conectores, pronombres, morfología verbal). Todos son mecanismos que los niños deben aprender a manejar para referirse a los objetos mencionados antes, omitir alguna información innecesaria, relacionar acontecimientos y progresar sin sobresaltos a través de la narración (Karmiloff \& Karmiloff-Smith, 2005). El presente estudio analiza dos aspectos importantes del desarrollo narrativo de manera interdependiente: la referencia a las personas y la estructura de contenido global.

\section{I.2.I. Referencia personal}

Esta dimensión referencial se vincula con un importante principio de cohesión narrativa: la marcación del estatus nuevo/dado de la información. La oposición nuevo/dado es un principio universal en la organización del discurso, que se expresa de diferentes maneras a través de las lenguas. Sin embargo, los estudios translingüísticos han señalado un buen número de vínculos sistemáticos entre el estatus cognitivo (nuevo/dado) de lo que se dice y las correspondientes formas lingüísticas en el sistema referencial (Gundel, Herberg \& Zakarski, 1993). Por ejemplo, los artículos indefinidos se usan para introducir referentes totalmente nuevos, los 
pronombres para aludir a un referente ya activado y los artículos definidos para un referente único e identificable. Asimismo, el orden sintáctico de los elementos de la oración es considerado un medio complementario (y más opcional) para marcar el estatus de la información en español. De este modo, los elementos ubicados en posición inicial de cláusula corresponden típicamente a la información conocida por los hablantes, y los que se presentan al final de la cláusula corresponden a la información nueva (Haviland \& Clark, 1974; Paprotté \& Sinha, 1987).

En cuanto al uso de los definidos, varios estudios sobre su naturaleza concluyen que no siempre se utilizan con la función descrita tradicionalmente, la de accesibilidad total de los referentes (Löbner, I 985; Fraurud, 1990; y para una revisión Recasens, Martí \& Taulé, 2009). Desde esta perspectiva, se afirma que muchos de los artículos definidos en español están gramaticalizados. Es el caso de aquellos incluidos en expresiones de alta frecuencia $\circ$ unidades de uso, cuyo núcleo nominal es un concepto funcional (como 'la prensa' o 'el correo') o bien relacional (como 'la esposa' o 'el tutor'), término que se define a partir de sus relaciones con otros objetos. Estos artículos definidos no aportan información semántica al concepto y suelen aparecer en las primeras menciones del referente. Los demás artículos definidos (no gramaticalizados) conservan el significado semántico de los núcleos nominales a los que determinan (como 'niño' o 'árbol'). A diferencia de los primeros, suelen aparecer solo en las menciones posteriores a la primera, en función de mantención referencial (cumplen, así, la función asignada por la aproximación tradicional). En esta investigación, se analizan principalmente los definidos en tanto marcadores del carácter 'dado' de los referentes, aunque también se observan casos de conceptos funcionales y su manifestación a través de las edades.

\section{I.2.2. Estructura global de contenido}

En cuanto a la estructura narrativa global, la investigación reciente ha mostrado una tendencia evolutiva infrecuente: la progresiva 'atenuación de la narratividad' (Berman \& Nir-Sagiv, 2004, 2007; Tolchinsky, 2007; Berman, 2008). Es infrecuente porque muestra un patrón de desempeño que decrece, a diferencia de todas las demás expresiones del desarrollo lingüístico observables desde los primeros meses de vida hasta bien entrada la infancia, alrededor de los 9 años.
Se aprecia que las narraciones a esta edad presentan un dominio correcto de la estructura narrativa en su sentido más canónico: historias con una situación inicial, una resolución final y fuertemente focalizadas en los eventos, en ocasiones incluso como una secuencia lineal de los mismos. En cambio, las narraciones de los adolescentes ( 16 años) y de los adultos se distancian de lo canónico y prototípico, incorporando formas lingüísticas propias del texto expositivo. Además, organizan la información en estructuras marcadas por las evaluaciones en lugar de los eventos (Tolchinsky, 2007).

La alta presencia de sucesiones de eventos en los relatos de los más jóvenes se ha descrito como una fase de procesamiento ascendente en el desarrollo de los géneros. Es un tipo de procesamiento que opera desde los datos concretos (los eventos, en este caso) hacia las generalizaciones. Gradualmente, este tipo de procesamiento se vuelve descendente y bidireccional (aproximadamente a los 16 años), regido más bien por las reflexiones y justificaciones de los sucesos (Berman, 2008). Habría, entonces, un cambio decisivo en el modo de concebir lo que es contar una historia: mientras los menores lo entienden como relatar una sucesión de eventos, los más grandes lo ven como una explicación de su propia versión y reflexión sobre los hechos (Tolchinsky, 2007).

\section{Metodología}

\section{I. Participantes}

La investigación analiza el discurso de 80 sujetos (40 hombres y 40 mujeres) de cuatro grupos de edad y niveles de escolarización (del sistema escolar español): primaria, (9-10 años, grupo identificado como PRI en los resultados), secundaria (I2I3, identificado como SEC), bachillerato (16-17, identificado como BAC), y adultos graduados (2230 , identificado como ADU).

Un criterio importante para determinar este rango etario se relaciona con la trayectoria evolutiva de los discursos. Estudios anteriores sobre desarrollo narrativo han demostrado que a los 9 años los niños han alcanzado un dominio estable de la estructura narrativa básica (Berman \& Slobin, 1994). Por ello, se considera esta edad como punto de partida para observar los cambios posteriores al logro de tal dominio. Por otro lado, los adultos debían cumplir con el requisito de haber escrito, al menos, un informe académico. En general, son estudiantes de 
postgrado en disciplinas de humanidades, ciencias sociales o ciencias exactas. Quedaron excluidos los estudiantes provenientes de formaciones en lengua y literatura.

Los 80 participantes son hablantes nativos del español peninsular, pertenecen a la región de Córdoba (España) y provienen de contextos familiares y escolares monolingües. Con el fin de estabilizar la variable sociocultural, se seleccionaron estudiantes de clase media, lo que garantizaba una procedencia familiar relativamente educada y cierta exposición a la lengua estándar tanto en el contexto escolar como familiar.

\subsection{Tareas}

Una meta central del estudio es conocer y comparar las habilidades de construcción global de los textos y sus formas de expresión lingüística en discursos extendidos. Se considera que estos constituyen el entorno adecuado para estudiar los desarrollos tardíos del lenguaje (Berman, 2008). Por ello, se pidió a los participantes que produjeran dos tipos de discurso (aunque esta investigación solo se aboca a uno de ellos) a partir de un video (detalles de la elicitación en el próximo apartado). El primero es la 'narración de experiencia personal', tarea en la que se pidió a los sujetos que contaran una historia oral y otra escrita acerca de algún conflicto interpersonal que ellos hubieran vivenciado. El segundo es la 'reflexión expositiva', en que se les pidió desarrollar el tema del conflicto interpersonal en una exposición oral y otra escrita.

Ambos tipos de tarea habían sido sometidas anteriormente a un extenso pilotaje (Aparici, Perera, Rosado \& Tolchinsky, 2000) el que reveló que, en estas condiciones, los sujetos no necesitan (ni disponen de) conocimiento mutuo con el interlocutor. Esto obliga a los hablantes a construir la cohesión de modo exclusivamente intralingüístico (y no deíctico), lo que constituye uno de los focos del estudio. Por otro lado, estas tareas pueden realizarse sobre la base de un mismo tema que resulta cercano y familiar para todos los estudiantes.Así, en ausencia del estímulo, incluso los participantes más jóvenes pueden producir los textos sin necesidad de hacer un excesivo esfuerzo de memoria.Además, el hecho de que se construyan acerca del mismo tema hace posible el control del contenido, lo que favorece la comparabilidad de los fenómenos lingüísticos.

Cada sujeto, entonces, produjo dos textos narrativos y dos textos expositivos. Se aplicó un orden contrabalanceado en la producción de los textos, de tal modo que la mitad de los participantes produjo en primer lugar las narraciones y en segundo lugar las reflexiones expositivas. Asimismo, la mitad de sujetos produjo los textos orales antes que los escritos y la otra mitad lo hizo a la inversa, con la finalidad de neutralizar el efecto del orden de aplicación en los resultados.

\subsection{Recolección, transcripción y procesamiento de datos}

Se dio a los participantes una introducción que los motivara y los orientara a la tarea de manera general. Se les pidió colaborar en un proyecto internacional para comparar cómo los niños y los jóvenes hablan y escriben acerca de determinados temas. El corpus empleado en este estudio forma parte de una muestra mayor de textos recogidos para el programa de investigación titulado Developing literacy in different contexts and in different languages (Berman \& Verhoeven, 2002; Berman, 2008). Este programa fue llevado a cabo en diversos países y siete lenguas (alemán, inglés, francés, hebreo, islandés, español peninsular y sueco)'.

Después de la introducción, se dijo a los participantes que verían un video breve que transcurre en una escuela y muestra distintos tipos de problemas. Se les dijo que ellos deberían luego hablar y escribir acerca de los 'problemas entre las personas'. El vídeo, con una duración de tres minutos y sin diálogos ni otro tipo de actividad verbal (pero con música) aborda varios tipos de conflicto que pueden darse en una institución educativa, tales como conflictos valóricos (personas que copian en un examen, por ejemplo), sociales (como el aislar a un compañero) y físicos (como peleas).

Para elicitar las narraciones, se les pidió que contaran una historia sobre algún incidente o situación en la que hubieran experimentado problemas con alguien. Se señaló explícitamente que no debían describir lo observado en el video sino contar una historia de algo experimentado personalmente. Para el texto expositivo, se les pidió tratar el tópico de los problemas entre las personas: dar una charla en clase y escribir una composición. Del mismo modo, se solicitó explícitamente no contar una historia, sino expresar sus ideas acerca del tema (más antecedentes sobre la elicitación en Berman \& Verhoeven, 2002). Este procedimiento -la presentación de un mismo estímulo para todos los sujetos- favorece también el control del contenido de las historias. Ello resulta 
importante para la comparación en el plano de la estructura y los mecanismos referenciales que se propone este trabajo.

Los textos fueron transcritos en formato $\mathrm{CHAT}^{2}$ (MacWhinney, 2000) y codificados con CLAN (Computerized Language Analysis) considerando algunas unidades de análisis relevantes para los objetivos de la investigación, tales como la cláusula. Así, cada cláusula ocupa una 'línea de texto', según las convenciones de transcripción de CHAT. La noción de cláusula con que se trabajó es similar a la oración simple de la gramática tradicional y corresponde a "todo predicado que describe una sola situación (una actividad, evento o estado)" (Berman \& Slobin, 1994:660).

El procesamiento de datos se hizo con pruebas ANOVA simples para determinar la significatividad estadística de las diferencias intragrupales y ANOVA de medidas repetidas para comparar desempeños orales y escritos. Además, se aplicaron pruebas chi cuadrado para procesar las variables discretas obtenidas a partir del análisis de mecanismos de referencia personal en los componentes de estructura narrativa. Todos los análisis estadísticos se hicieron con el programa SPSS versión 16 para Macintosh.

\section{Resultados}

\section{I. Estructura narrativa}

Siguiendo el trabajo pionero de Labov y Waletzky (1967) y Labov (1972), los componentes de la estructura narrativa analizados son: resumen (breve aclaración inicial de lo que se tratará la historia), orientación (definición de la situación inicial, con marco temporal, espacial y presentación de personajes), complicación (episodio clave), episodios (otros eventos que no constituyen complicación pero que hacen avanzar la historia), resolución (solución o desenlace), evaluación (reflexión del narrador), coda (cierre) y partes sin estructura narrativa (SEN).

Los ejemplos (I) y (2) ilustran algunas de las diferencias que se dan entre los grupos respecto de la estructura narrativa, cuyo análisis se muestra a la derecha del texto, en la segunda columna. Se puede observar cómo en (I), que pertenece al grupo más joven, hay gran cantidad de fragmentos típicamente narrativos en proporción al número total de cláusulas (complicación y episodios). En cambio, en (2), que pertenece al grupo de adultos, si bien hay muchos episodios (es un texto que tiene bastantes

(I) Primaria, escrito:

\begin{tabular}{|l|l|l|}
\hline Un dia yo me pelee con un compañero. & RESUMEN & I \\
\hline Empezó el, le escupio a mi amigo & COMPL. & 2 \\
\hline y sin hacerle nada. & EVALUACIÓN & I \\
\hline $\begin{array}{l}\text { Después, en la clase que hay por la tarde, mi amigo dijo que le pegáramos y yo } \\
\text { le dije que no. }\end{array}$ & EPISODIO I & 2 \\
\hline $\begin{array}{l}\text { Después me dijo Abel, que es que el que empezo a escupirle a Alberto, me dijo } \\
\text { "cuando salgamos te voy a pegar". Me lo dijo a mí. }\end{array}$ & EPISODIO 2 & 4 \\
\hline $\begin{array}{l}\text { Al terminar la clase no me pego pero mi amigo Alberto le escupio y le estaba } \\
\text { pegando patadas a la cartera }\end{array}$ & EPISODIO 3 & 3 \\
\hline $\begin{array}{l}\text { y despues nos dijo a los dos una palabrota muy gorda dige yo "ami, ami” y le } \\
\text { meti una patada y despues le coji de los pelos y lo lleve asta el suelo. }\end{array}$ & EPISODIO 4 & 5 \\
\hline Se fue y me segía insultando. & EPISODIO 5 & 2 \\
\hline Yo lo deje y me fui a mi casa. & RESOLUCIÓN & 2 \\
\hline $\begin{array}{l}\text { A ese niño le he pegado muchas veces por otras cosas. } \\
\text { (pgIOmnw) }\end{array}$ & CODA & I \\
\hline TOTAL DE CLÁUSULAS & & $\mathbf{2 3}$ \\
\hline
\end{tabular}


(2) Adultos, escrito:

\begin{tabular}{|l|l|l|}
\hline $\begin{array}{l}\text { El caso que mejor recuerdo de todos aquellos que un niño vive en su infancia } \\
\text { me sucedió en lo que antes se llamaba Educación General Básica, más } \\
\text { concretamente en primero. }\end{array}$ & RESUMEN & 4 \\
\hline $\begin{array}{l}\text { Mis especiales cualidades, al ingresar en este colegio y en aquel curso, fueron } \\
\text { que no lo hice en su momento (en septiembre) sino en el mes de enero. El } \\
\text { porqué no es otro que yo fui adoptado por mis padres a la edad de seis años. } \\
\text { Durante ese transcurso de tiempo no había recibido ninguna escolarización... }\end{array}$ & ORIENTACIÓN & 5 \\
\hline $\begin{array}{l}\text { con lo cual en pleno mes de enero y con el retraso educativo que arrastraba } \\
\text { lo primero que sufrí fue rechazo. }\end{array}$ & EPISODIO I & 3 \\
\hline $\begin{array}{l}\text { No voy a entrar en pormenores del porqué esa súbita escolarización... sino } \\
\text { en el rechazo de mis compañeros.La verdad es que yo no podía comprender } \\
\text { el porqué de aquella actitud conmigo. Simplemente melimitaba a sufrir } \\
\text { aquellos ademanes para con mi persona. }\end{array}$ & EVALUACIÓN & 6 \\
\hline $\begin{array}{l}\text { Recuerdo muy nítidamente como una vez, en plena clase, uno de mis } \\
\text { compañeros -Emilio- había traido un coche de plástico para jugar en el } \\
\text { recreo. }\end{array}$ & EPISODIO 2 & \\
\hline Yo me había fijado en él. Era un coche rojo precioso. & & 3 \\
\hline $\begin{array}{l}\text { Me acerqué y le dije si podíamos jugar juntos, con su coche durante el recreo. } \\
\text { El me respondió que sí }\end{array}$ & EPISODIO 3 & 4 \\
\hline pero cuál fue mi sorpresa & EVALUACIÓN & I \\
\hline que aquello nunca se produjo. & COMPLICACIÓN & I \\
\hline $\begin{array}{l}\text { Llegado el recreo, mis ánimos se encendieron pensando que iba a jugar con } \\
\text { aquel coche. }\end{array}$ & EPISODIO 4 & 2 \\
\hline Pero Emilio pensó que yo no era el apropiado o iqué sé yo! & EVALUACIÓN & 3 \\
\hline $\begin{array}{l}\text { La verdad es que él se encontraba jugando con otros niños y yo me quedé } \\
\text { fuera. }\end{array}$ & EPISODIO 5 & 4 \\
\hline $\begin{array}{l}\text { Como aquel caso recuerdo varios en aquel fatídico año pero precisamente } \\
\text { eqte que acabo de contar me impactó muchísimo. Es posible que fruto de } \\
\text { aquella frustración en mí se generará una atracción muy fuerte por todo lo } \\
\text { quenga de los coches. }\end{array}$ & EVALUACIÓN & 6 \\
\hline $\begin{array}{l}\text { Atracción y gusto que aún hoy siguen perdurando con lo cual a lo mejor } \\
\text { tengo que darle las gracias a Emilio. } \\
\text { (pu07mnw) }\end{array}$ & CODA & \\
\hline TOTAL DE CLÁUSULAS & & \\
\hline
\end{tabular}


Tabla I. Análisis de la distribución de los componentes de la estructura narrativa en los ejemplos (I) y (2).

\begin{tabular}{|l|rr|rr|}
\cline { 2 - 5 } \multicolumn{1}{c|}{} & \multicolumn{2}{c|}{ Texto I (primaria) 23 cláusulas } & \multicolumn{2}{c|}{ Texto 2 (adultos) 46 cláusulas } \\
\cline { 2 - 6 } \multicolumn{1}{c|}{} & Recuento & Porcentaje sobre el $\mathrm{n}^{\circ}$ de cláusulas & \multicolumn{1}{c|}{ Recuento } & Porcentaje sobre el $\mathrm{n}^{\circ}$ de cláusulas \\
\hline Resumen & $\mathrm{I}$ & 4,34 & 4 & 8,69 \\
Orientación & 0 & 0 & 5 & 10,86 \\
Complicación & 2 & 8,70 & 1 & 2,17 \\
Evaluación & 1 & 4,34 & 18 & 39,13 \\
Episodios & 16 & 69,56 & 16 & 34,78 \\
Resolución & 2 & 8,70 & 0 & 0 \\
Coda & 1 & 4,34 & 2 & 4,34 \\
\hline TOTAL & 23 & 100 & 46 & 100 \\
\hline
\end{tabular}

más episodios narrativos que la media de su grupo), también abundan las cláusulas evaluativas. Después de los ejemplos se presenta un resumen de este análisis en la Tabla I.

Tal como se muestra en los ejemplos (I) y (2) y en la Tabla I, un primer resultado sobre la estructura es la evidencia de que la presencia de los componentes eventivos (complicación y episodios) de la narración disminuyen con la edad. Los dos grupos de sujetos más jóvenes, como puede observarse en el Gráfico I, construyen sus narraciones principalmente sobre la base de episodios (EPI); más del $30 \%$ de las cláusulas de sus textos corresponden a este componente. En segundo lugar, los jóvenes prefieren la complicación (CO). Ambos componentes dan a los textos su carácter más típicamente narrativo: contienen acciones y sucesos concretos que hacen avanzar la historia.

Del mismo modo, se evidencia que los componentes evaluativos de la narración aumentan con la edad. Los dos grupos de sujetos mayores construyen sus narraciones principalmente sobre la base del componente evaluación (EV); más del $25 \%$ de las cláusulas del texto corresponden a este componente (Gráfico I). Las cláusulas evaluativas transmiten la perspectiva de un narrador que puede distanciarse y adoptar una determinada actitud respecto de lo que cuenta. Contribuyen, además, a dar sentido al relato y valorar sus contenidos en distintos grados. También se observa en los grupos mayores una mayor diversidad estructural: más componentes y uso menos canónico de los mismos.

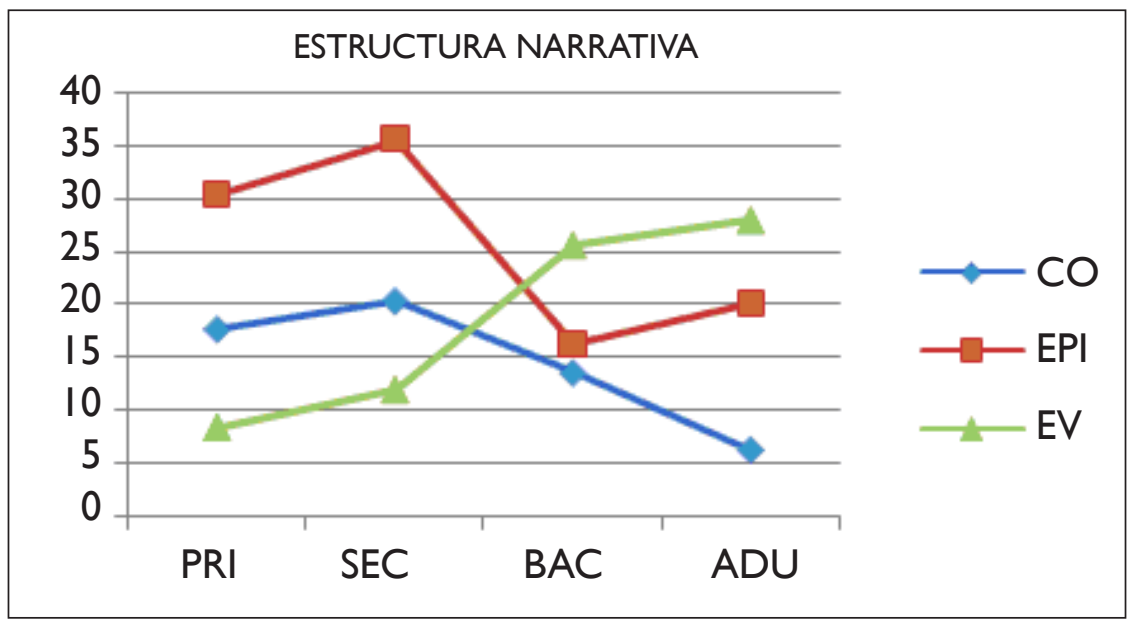

Gráfico I. Distribución de los tres principales componentes de la estructura narrativa en los textos orales de los cuatro grupos. Porcentajes sobre el total de cláusulas por texto. 
Tabla 2. Análisis de la distribución general de referentes de persona que se introducen y mantienen en los ejemplos (I) y (2).

\begin{tabular}{|l|c|c|}
\cline { 2 - 3 } \multicolumn{1}{c|}{} & Texto I (primaria) & Texto 2 (bachillerato) \\
\hline Cláusulas & 21 & 40 \\
\hline Referentes nuevos & 8 & 5 \\
\hline Mantenciones referenciales & 19 & 48 \\
\hline $\begin{array}{l}\text { Proporción de referentes } \\
\text { nuevos por cláusula }\end{array}$ & $\begin{array}{c}0,38(8 / 2 I)=\text { I referente nuevo cada } \\
2,63 \text { cláusulas }\end{array}$ & $\begin{array}{c}0,12(5 / 40)=\text { I referente nuevo cada } \\
8,33 \text { cláusulas }\end{array}$ \\
\hline $\begin{array}{l}\text { Proporción de mantenciones } \\
\text { por referente }\end{array}$ & $\begin{array}{c}2,37(19 / 8)=\text { cada referente nuevo } \\
\text { reaparece 2,37 veces }\end{array}$ & $\begin{array}{c}9,6(48 / 5)=\text { cada referente nuevo } \\
\text { reaparece } 9,6 \text { veces }\end{array}$ \\
\hline
\end{tabular}

A tal punto es así, que algunos textos de los adultos podrían considerarse no narrativos, debido a que se construyen fundamentalmente sobre la base de reflexiones, con rasgos de texto expositivo.

La prueba ANOVA permite comprobar el efecto de la edad en el uso que se hace de los componentes episodios y complicación, ya que las diferencias entre los grupos resultan significativas en las dos modalidades (episodios, oral $F(3,76)=4.402, p$. < .007 y escrita $F(3,76)=6.800, p .<.000$; complicación, oral $F(3,76)=4.443, p .<.006$ y escrita $F(3,76)=$ $4.079, p .<.010)$. La prueba ANOVA de medidas repetidas, en cambio, informa que no hay efecto de la modalidad para estas variables. Asimismo, las pruebas estadísticas muestran que el efecto de la edad es significativo en las dos modalidades para el componente evaluativo (oral $F(3,76)=5.952$, $p$. $<.001$ y escrita $F(3,76)=4.955, p .<.003)$ y que tampoco hay efecto de la modalidad para esta variable $F(I, 76)=2.674, p .<.106$. No obstante esto último, vale destacar que, en general, aumenta la presencia de episodios, orientación y evaluación en las narraciones escritas, es decir, aumenta la información propiamente narrativa, por oposición a la información no narrativa (codas, discurso directo, etc.).

\subsection{Referencia personal}

\subsection{Introducción y mantención de la referencia}

El análisis de la referencia personal incluye todas aquellas expresiones que aluden a las personas $\mathrm{u}$ otras entidades animadas (como la mascota, la familia o la iglesia) dentro de la narración. La introducción de referentes personales corresponde a aquellos referentes que se mencionan por primera vez en el texto y su proporción se calcula en función del número de cláusulas del texto. La mantención de referentes personales corresponde a todas las menciones referenciales posteriores a la primera y se calcula en función del número de referentes nuevos del texto.

En los ejemplos (3) y (4) todos los referentes que se mencionan por primera vez en el texto se han destacado y numerado con negrita para efectos del análisis. Y las mantenciones, es decir, todas las apariciones de un referente en el texto después de la primera, se han destacado y numerado en cursiva. El uso de [ ] y mayúsculas señala la presencia de referentes marcados originalmente solo en la desinencia verbal (elementos cero). La síntesis de este análisis se presenta a continuación de los ejemplos, en la Tabla 2.

\section{(3) Primaria, oral:}

Bueno, todo empezó cuando nosotros (I) estábamos dando un paseo. Entonces [YO (2)] vi para atrás y $[Y O(I)]$ vi a tres niños (3), uno rubio y dos morenos. Eh... entonces [NOSOTROS (2)] nos fuimos a la piscina de arena saltando y luego eh... Ricardo (4) cayó en la arena. Y luego [ELLOS (3)] le [RICARDO (4)] echaron un escupitajo por atrás. Eh... entonces [NOSOTROS (5)] nos chivamos a la señorita (5) entonces la señorita (6) no les (7) dijo nada.

Luego al otro día [NOSOTROS (8)] nos chivamos otra vez y no les (9) pasó nada y al último día [NOSOTROS(I0)] nos chivamos a un profesor de inglés (6) y los (I I) castigaron (7) ahí.

Entonces ¿cómo era ? Bueno ahí entonces, al otro día ya [ELLOS (I2)] no nos (I3) hicieron nada. Pero al otro eh... [ELLOS (I4)] se chivaron y nosotros (I5) no estábamos haciendo nada y [NOSOTROS (I6)] nos tuvimos que sentar al lado de un profesor 
(8) entonces al otro día [NOSOTROS (I 7)] les (I8) tiramos piedras y de todo por chivarn... porque [ELLOS (19)] se chiven. Entonces ya acabó ahí.

(pgl5mns)

(4) Bachillerato, oral:

María (I) era una chica que estaba [MARÍA (I)] en el colegio pero [LOS DEMÁS, SUS COMPAÑEROS (2)] no la(2) querían mucho porque aparte de que [MARÍA (3)] era fea [MARÍA (4)] hablaba muy raro y [MARÍA (5)] no suele estar mucho con la gente (6) y [ELLOS (7)]la (8) tenían discriminada, entre comillas. Entonces una vez ya a finales de curso María, (9) que no le caían muy bien sus compañeros, (I0) dijo que... [MARÍA (I I)] dijo a su tutor (3) que le habían... que le (12) habían hecho unos mo... moratones en las piernas sus compañeras de clase (4). Y claro, el profesor (I3)le (I4) dijo que se (I5) lo enseñara [MARÍA (I6)] porque aunque ella (I7) era muy callada en clase y [MARÍA (I8)] era muy buena era muy difícil que sus compañeras (19)le (20) pegaran patadas. Y ella (2I) dijo que no, que es que como [MARÍA (22)] las tenía por encima de la pierna, por el muslo pues claro como [MARÍA (23)] llevaba vaqueros no se (24) lo podía enseñar [MARÍA (25)]. Entonces sus compañeros (26) se alejaron más de ella (27).Ella (28) estaba sola en los recreos. Hasta que un día una chica que le daba pena (5) [LA CHICA (29)] se acercó a ella (30) y claro como María (3I) se confiaba mucho cada vez que se a cada vez que alguien se le (32) acercaba [MARÍA (33)] le contó

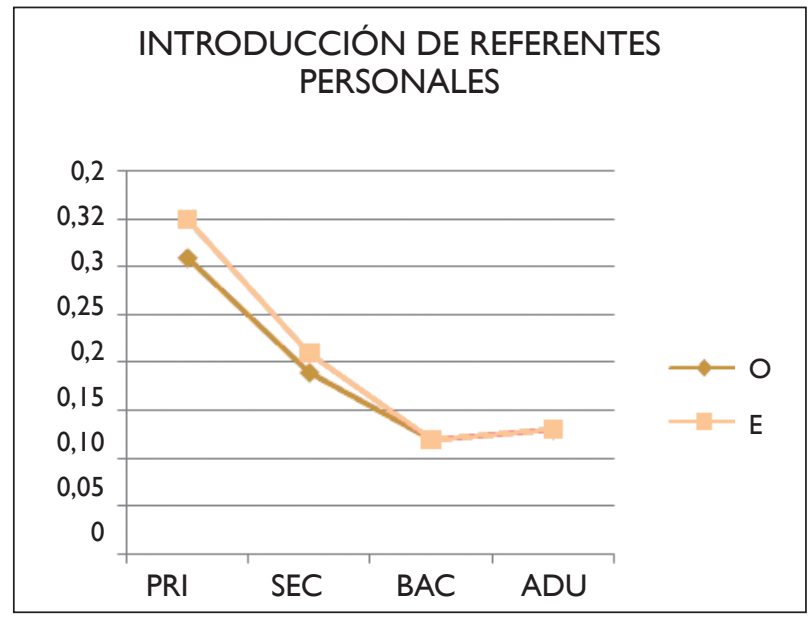

Gráfico 2. Introducción de referentes, modalidades oral y escrita. Media de proporciones sobre el número de cláusulas. que todo era mentira. Que ella no que no le (34) habían dado patadas ni nada [LAS COMPAÑERAS (35)]. Ella (36) en cuanto se lo contó María (37) fue a contárselo a sus compañeras (38) y ellas (39) no se sorprendieron porque claro, porque ellas (40) sabían que era mentira y lo que [LAS COMPAÑERAS (4I)] esperaban era que María (42) se lo contara al tutor (43) pero como [MARÍA (44)] no lo hizo la chica (45) que se le (46) había acercado ya no habló más con ella (47). Se alejaron todos (8) y terminó el curso. Y ya está.

(ph08fns)

Como se desprende de los análisis expuestos, la tasa de introducción referencial disminuye notablemente con la edad. Los grupos de sujetos menores, tal como se ilustra en el Gráfico 2, presentan una proporción alta de referentes nuevos (modalidad oral: primaria 0,31 y secundaria 0,19). Es decir, los textos de primaria presentan una media de referente nuevo cada 3 cláusulas y los de secundaria presentan una media de I referente nuevo cada 5 cláusulas. Los grupos de sujetos mayores exhiben una proporción baja de referentes nuevos (modalidad oral: bachillerato 0,12 y adultos 0,13 ). Es decir, estos sujetos introducen, en promedio, I referente nuevo cada 8 cláusulas (Gráfico 2). A la inversa y como también se desprende de los ejemplos anteriores, la tasa de mantención referencial aumenta notablemente con la edad. Los grupos de sujetos menores, como se ilustra en el Gráfico 3, presentan una proporción baja de mantención referencial (primaria 4,8I y secundaria 6,I3). Es decir, hay entre

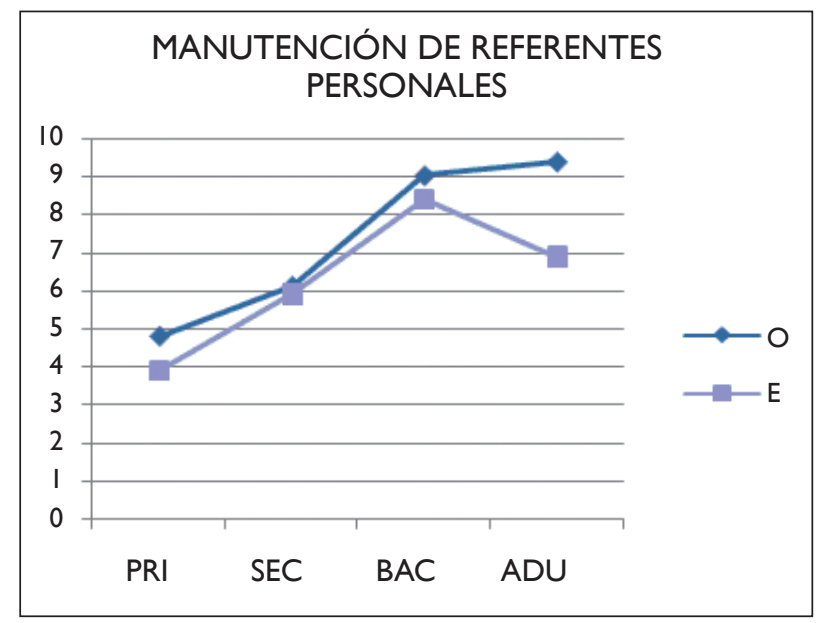

Gráfico 3. Mantención de referentes, modalidades oral y escrita. Media de proporciones sobre el total de referentes introducidos. 
5 y 6 'mantenciones' (reapariciones del referente) por cada uno de los referentes introducidos. Los grupos de sujetos mayores exhiben una proporción alta: bachillerato 9,05 y adultos $9,4 \mathrm{I}$, es decir, hay más de 9 mantenciones por cada nuevo referente del discurso. En la modalidad escrita, las diferencias entre los grupos son similares a las de la modalidad oral, aunque las medias de todos los grupos descienden un poco.

En consecuencia, las diferencias entre los grupos son altamente significativas para la introducción referencial (modalidad oral $\mathrm{F}(3,73)=9.07 \mathrm{I}, \mathrm{p}$. < .000 , y modalidad escrita $F(3,73)=|3.59|$, p. $<.000)$, es decir, se verifica el efecto de la edad en ambas modalidades. También se comprobó que todos los grupos, salvo el de adultos, introducen más referentes cuando escriben y consecuentemente se verifica estadísticamente el efecto de la modalidad $(\mathrm{F}(\mathrm{I}, 73)=4.827, \mathrm{p} .<.03 \mathrm{I})$. En cuanto a la mantención, las diferencias entre los grupos también son significativas en ambas modalidades (oral $\mathrm{F}(3$, $73)=5.476, p .<.002$, y escrita $F(3,73)=5.802$, p. $<.00 \mathrm{I})$, y las diferencias entre ambas modalidades nuevamente resultan significativas $F(I, 73)=|7.29|$, p. $<.000)$ e interactúan $F(I, 73)=4.389$, p. $<.007$. La modalidad afecta de modo tal que todos los grupos disminuyen la tasa de mantención referencial en sus textos escritos.
3.2.2. Formas lingüísticas y funciones de la referencia personal

Otro resultado destacable en el plano de la referencia personal se relaciona con la habilidad para utilizar formas lingüísticas que establecen el estatus nuevo/dado de la información. Las formas lingüísticas analizadas en la referencia personal son: sintagma nominal definido e indefinido, pronombre y marca morfológica (elemento cero). Los sujetos menores, como se observa en el Gráfico 4, y particularmente los de primaria, utilizan un número elevado de sintagmas nominales definidos para introducir los referentes de persona y un número relativamente bajo de sintagmas nominales con determinante indefinido para hacerlo. Esto no significa que cometan transgresiones graves en la marcación del estatus de la información, aunque sí algunas ambigüedades. Además, prefieren aludir frecuentemente a referentes supuestamente conocidos por el interlocutor o que ostentan roles conocidos como 'el tutor', 'el director'. Ello con la finalidad de no tener que alternar formas definidas con indefinidas a lo largo del texto. Los sujetos mayores, en tanto, prefieren notablemente los sintagmas indefinidos para introducir los referentes (Gráfico 4) y muestran más habilidad para alternar el uso de indefinidos y definidos con distintas funciones en el discurso. En consecuencia, las diferencias entre

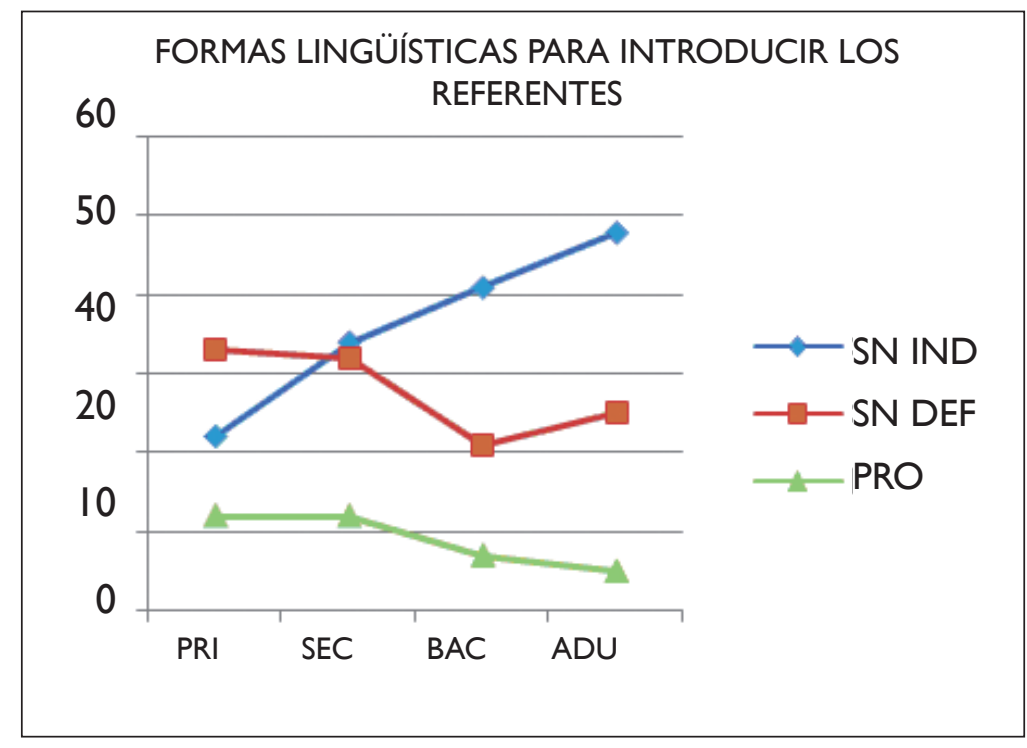

Gráfico 4. Principales formas para la introducción de referentes, modalidad oral. Porcentajes sobre el total de referentes introducidos. 
los grupos son estadísticamente significativas para el uso de determinantes indefinidos en las dos modalidades (modalidad oral $\mathrm{F}(3,73)=3.008$, p. $<$ .036 y modalidad escrita $F(3,73)=5.059$, p. $<.003)$ y no se observa efecto de la modalidad.

\subsection{Referencia personal y estructura narrativa}

En consonancia con los resultados anteriores, se encontró que los sujetos más jóvenes, tal como se aprecia en el Gráfico 5, introducen sus referentes personales principalmente en los componentes de complicación y episodios. Los sujetos mayores, en cambio, prefieren introducir los referentes en la orientación y la evaluación. En cuanto a la mantención, los más jóvenes mantienen la referencia personal en los episodios, como muestra el Gráfico 6, con mucha diferencia respecto de los otros componentes. Los adolescentes de 16 años mantienen la referencia tanto en los episodios como en la evaluación, mientras que los adultos prefieren la evaluación, también con mucha diferencia. En segundo lugar, este grupo mantiene la referencia en los episodios, y en tercer lugar ocupan la orientación, componente

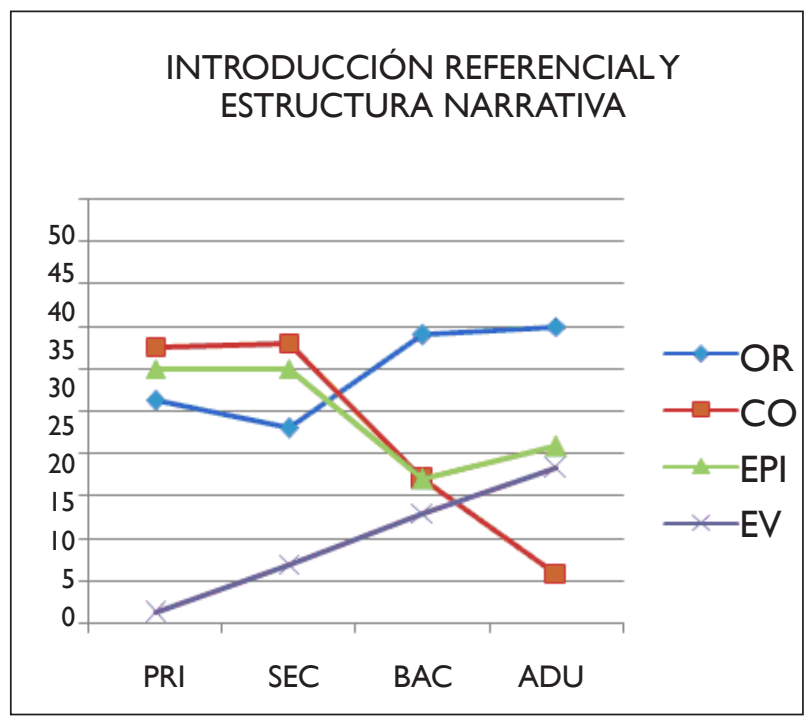

Gráfico 5. Introducción de referentes en los componentes principales de la estructura narrativa, modalidad oral. Porcentajes sobre el total de referentes que se introducen por grupo. que no parece relevante en ningún otro grupo para mantener los referentes. Ello muestra una diferencia del grupo adulto respecto de todos los demás, que estaría relacionada con el mayor 'tiempo textual' que destinan a la planificación y a los preámbulos de la historia.

En suma, la distribución de la introducción y la mantención de referentes personales evoluciona desde los componentes eventivos hacia los componentes orientativos (para la introducción) y evaluativos (para la introducción y la mantención).

Para los dos resultados antes comentados el grado de asociación entre las variables resulta significativo en todos los grupos y las dos modalidades, primaria oral y escrito $\chi^{2}(6, N=358)=65.542 \mathrm{p}=0.000 ; \chi^{2}(7$, $\mathrm{N}=319)=89.354 \mathrm{p}=0.000$; secundaria oral $y$ escrito $\chi^{2}(4, \mathrm{~N}=609)=59.783 \mathrm{p}=0.000 ; \chi^{2}(6, \mathrm{~N}=555)=$ $46.436 \mathrm{p}=0.000$; bachillerato oral y escrito $\chi^{2}(7$, $\mathrm{N}=953)=1 \mid 4.006 \mathrm{p}=0.000 ; \chi^{2}(7, \mathrm{~N}=8 \mid 8)=85.645$ $\mathrm{p}=0.000$; adultos oral y escrito $\chi^{2}(7, \mathrm{~N}=1399)=$ $59.068 \mathrm{p}=0.000 ; \chi^{2}(7, \mathrm{~N}=975)=49.489 \mathrm{p}=0.000$. Las diferencias entre modalidades no resultan relevantes.

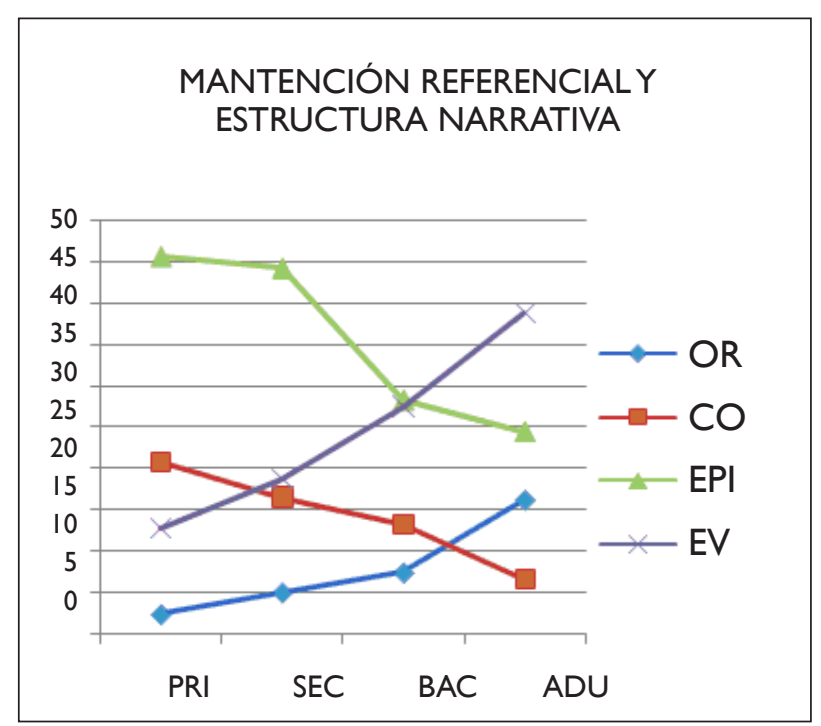

Gráfico 6. Mantención de referentes, modalidades oral y escrita. Media de proporciones sobre el total de referentes introducidos. 


\section{CONCLUSIONES}

Muchos de los resultados obtenidos pueden interpretarse como el producto de diferentes modos de procesar la información en la producción del discurso, modos que se relacionan significativamente con la edad y la escolarización.

Los más jóvenes están preferentemente guiados por el nivel oracional y organizan la información a medida que van hablando. Esto se comprueba en las tres dimensiones presentadas antes: I) La organización narrativa se realiza sobre la base de eventos y está dirigida, entonces, por un procesamiento de tipo ascendente. 2) Hay una alta tasa de introducción referencial, dirigida por el plano oracional a tal punto de introducir casi un referente por cláusula. También se observa dificultad (particularmente en el grupo más joven) para distinguir lingüísticamente el estatus nuevo/dado de la información (preferencia generalizada de sintagmas nominales definidos para funciones diferentes). Lo anterior comprueba que la gestión propiamente discursiva de tales formas se adquiere tarde, sobre los 10 años. Adicionalmente, estos grupos muestran una baja tasa de mantención referencial, lo que indicaría menos recursos lingüísticos y cognitivos para organizar los referentes en un plano supraoracional. 3) Los componentes de la estructura narrativa se utilizan con funciones poco diferenciadas entre sí (se introducen y se mantienen los referentes en los mismos componentes estructurales). Este rasgo señala poca especificidad y variedad en el uso que hacen de las distintas funciones narrativas.

Los mayores se orientan por el nivel discursivo, de modo tal que planifican y organizan eficazmente la información con perspectiva, capacidad de reflexión y opciones lingüísticas propias. Todo lo anterior también se verifica en las tres dimensiones comentadas: I) La organización narrativa destaca por la presencia de evaluaciones y está dirigida, en consecuencia, por un procesamiento descendente que permite a los hablantes reflexionar sobre los sucesos que van a contar e interpretarlos. 2) Hay una baja tasa de introducción referencial porque los sujetos disponen de un modelo del texto organizado con antelación. Además, dedican más tiempo textual a elaborar la información introducida y a volver sobre ella con descripciones e interpretaciones. Ello explica la alta tasa de mantención referencial y hace poco probable aumentar la cantidad de información nueva. Por otro lado, entre los mayores se observa un uso de formas lingüísticas variadas y específicas para marcar el estatus de la información (determinantes definidos/indefinidos). 3) Los componentes de la estructura narrativa se utilizan con funciones claramente diferenciadas y específicas para la gestión de los referentes de persona (se introducen y se mantienen los referentes en distintos componentes estructurales).

En cambio, no se registran mayores efectos de la modalidad en lo relativo a las funciones de la estructura narrativa, lo que se puede interpretar como una característica del género. Al respecto, se comprueba que, para el género narrativo, las diferencias entre las modalidades son menores que para otros géneros, como sugieren algunos estudios (Berman \& Ravid, 2009). El efecto de la modalidad sí resulta significativo en algunos de los fenómenos vinculados a la referencia personal: tasa de introducción y de mantención (todos introducen más y mantienen menos referentes personales cuando escriben) y uso de ciertas formas lingüísticas para mantener los referentes (todos utilizan más sintagmas nominales cuando escriben salvo los adultos). En suma, el efecto de la modalidad, en las edades y el género discursivo estudiado, está relacionado con los recursos lingüísticos y textuales, más que con lo relativo al contenido global del discurso.

En el caso de los cambios que experimenta la estructura narrativa, los resultados de la presente investigación coinciden con hallazgos anteriores. Se ha visto, por ejemplo, que los textos de los más jóvenes focalizan en la estructura eventiva. En cambio, los textos de los mayores están más dirigidos por las interpretaciones de los hechos (Tolchinsky, Rosado, Aparici \& Perera, 2005; Berman \& Nir-Sagiv, 2007; Tolchinsky, 2007; Berman, 2008). También la variabilidad intragrupal observada confirma resultados de otros estudios, por ejemplo, aquellos sobre desarrollo narrativo temprano (McCabe \& Peterson, 1991; Berman \& Slobin, 1994; Berman, 1997). Estos trabajos demostraron que los niños preescolares más jóvenes presentan una mayor variabilidad intragrupal respecto de diversas características de su producción discursiva (extensión, contenidos, estructura). Posteriormente, después de varios años de escolaridad, las diferencias intragrupales disminuyen para volver a aparecer en la adolescencia, como se ha comprobado en 
esta investigación. Además, los sujetos jóvenes se orientan más por los requerimientos de la construcción oracional que discursiva, como también se ha visto en estudios anteriores (Karmiloff-Smith, 1985; Hickmann, 2003). En cambio, los jóvenes de 16 años y los adultos narran con perspectiva, lo que se ve reflejado en la actitud epistémica que suelen adoptar y la tendencia a hacer conclusiones mediante generalización (Berman, Ragnarsdóttir \& Strömqvist, 2004).

En el caso de los referentes de persona, la alta tasa de mantención entre los mayores también dialoga con conclusiones anteriores sobre el desarrollo narrativo. Mantener la referencia es condición lingüística obligada para hacer descripciones de personajes, situarlos en el tiempo y en el espacio, hacer comentarios sobre sus acciones $y$, sobre todo, interpretar sus estados mentales. Todos estos fenómenos se han descrito teóricamente como logros evolutivos cruciales del desarrollo narrativo (Bruner, I 986; Ravid \& Berman, 2006). En cuanto a las formas de la referencia personal, nuestros resultados coinciden con otros estudios en el sentido de que el dominio intradiscursivo de la referencia se afianza hacia los 9 o 10 años (Karmiloff-Smith, 1985; Hickmann, 1995; Berman \& Katzenberger, 1998; Vion \& Colas, 1999).

A continuación se comentan brevemente (por razones de espacio) algunas de las proyecciones de la investigación. En primer lugar, sería interesante llevar a cabo estudios que aborden en profundidad el desarrollo tardío de otros géneros discursivos en las dos modalidades. De este modo, se dispondría de un repertorio más amplio de las formas propias de estas edades en relación con las funciones que imponen los distintos géneros. Si bien se cuenta con bastante información acerca de las formas lingüísticas que se incrementan en estas edades, todavía se conoce poco acerca de cómo estas son desplegadas en respuesta a las demandas de uso de los tipos de texto y las modalidades discursivas. Tales estudios resultan tanto más necesarios en una lengua como el castellano, que ha sido poco estudiada.

Lo anterior supone, por un lado, un conocimiento teórico relevante acerca de cómo el discurso se construye evolutivamente después del término de la infancia, una vez que las habilidades de base ya están adquiridas. Estudios anteriores (Berman \& Slobin, 1994; Berman, 1997) demostraron que esta competencia comienza con una alta divergencia individual muy temprana por la falta del dominio discursivo más elemental. Luego, deriva en un desempeño estable y homogéneo de las habilidades discursivas. Estas últimas se despliegan, hacia los 9 10 años, en construcciones textuales 'bien formadas', claramente identificables en su género y construidas sobre principios dicotómicos respecto de otros géneros (Berman \& Nir-Sagiv, 2007; Berman, 2008).

Particularmente importante sería poder proyectar estudios similares que permitan comparar estas competencias narrativas de base y las que se desarrollan posteriormente con las de niños y jóvenes provenientes de contextos desfavorecidos culturalmente. Este estudio pretende contribuir a situar un punto de partida desde el cual estudiar el desarrollo discursivo. En este sentido, queda todavía mucho por investigar para conocer los límites y los ritmos de los desarrollos tardíos del lenguaje en relación con sus determinantes culturales. Sin duda, estas últimas, vinculadas con factores tales como calidad de la escolaridad y capital cultural de las familias, podrían modificar sustantivamente los resultados de la presente investigación.

Otras proyecciones son posibles en el campo de la enseñanza del castellano como lengua materna, por ejemplo, aquellas relacionadas con ciertos rasgos en el desarrollo de las habilidades discursivas aquí estudiadas. Se destaca, entre ellos, la dificultad de los niños ( 9 años) para elaborar, durante la producción oral, la información acerca de los personajes que introducen en sus historias. Asimismo, su tendencia a focalizarse en los eventos o la limitada capacidad que demuestran todavía para marcar eficazmente el estatus de la información. Desde el punto de vista estructural, también se vislumbran algunas señales que pueden ser útiles para el sistema escolar. La primera es la necesidad de fomentar, desde los 10 años, habilidades narrativas crecientemente complejas y especializadas, tales como la descripción de espacios y la interpretación de los hechos, más que su mera exposición sumativa. La segunda es la conveniencia de articular un trabajo conjunto con otros géneros más desafiantes, como el expositivo por ejemplo, que ha demostrado ser un entorno propicio para el uso de un lenguaje rico y complejo (Tolchinsky et al., 2005; Berman \& Ravid, 2009).

En esta dirección, los resultados del presente trabajo también podrían contribuir al establecimiento de criterios claros y sistemáticos tanto para la evaluación como para la enseñanza de lo que debieran lograr 
los niños en determinados niveles de Enseñanza Básica y Media con sus producciones narrativas. Estos criterios podrían delimitarse para los textos tanto escritos como orales, contribuyendo así, desde la investigación, a un ámbito de la enseñanza poco atendido en Chile.También, tales criterios para la enseñanza y la evaluación de algunas habilidades discursivas podrían contribuir a neutralizar el efecto negativo de algunos factores socioculturales en los desarrollos tardíos del lenguaje.

Por último, esta investigación y otras en la misma línea, pueden mostrar la necesidad de trabajar las habilidades de comunicación en torno a los géneros y las modalidades del discurso. Este tipo de trabajo tiene como base la idea del desarrollo lingüístico como un proceso prolongado determinado por la evolución de las habilidades discursivas. Una creciente experiencia lingüística y letrada de calidad debería conducir a los hablantes desde un conocimiento esquemático de los géneros hacia el dominio competente, flexible y autónomo de los mismos, como rasgos continuos de la actividad comunicativa. 


\section{REFERENCIAS BIBLIOGRÁFICAS}

Aparici, M., Perera, J., Rosado, E. \& Tolchinsky, L. (2000). (Eds.). Developingliteracy across genres, modalities, and languages. Barcelona: Institute of Educational Sciences, University of Barcelona.

Berman, R. (1997). Developing form/function relations in narrative texts. Lenguas Modernas, 24, 45-60.

Berman, R. (2004). Between emergency and mastery:The long developmental route of language acquisition. En R. Berman (Ed.), Language development across childhood and adolescence (pp. 9-34). Amsterdam: John Benjamins.

Berman, R. (2008). The psycholinguistics of developing text construction. Journal of Child Language, 35, 735-77I.

Berman, R. \& Slobin, D. (1994). Relating events in earrative. A crosslinguistic developmental study. Hillsdale: Erlbaum.

Berman, R.\& Katzenberger, I. (1998). Cognitive and linguistic factors in development of pictureseries narration. Organization of Learners'Texts. Special Issue of Studi italiani di linguistica teorica ed applicata, 27, 21 -47.

Berman, R. \& Verhoeven, L. (2002). Cross-linguistic perspectives on the development of textproduction abilities: Speech and writing. Written Language and Literacy, 5, I-44.

Berman, R. \& Nir-Sagiv, B. (2004). Linguistic indicators of inter genre differentiation in later language development. Journal of Child Language, 31,339-380.

Berman, R., Ragnarsdóttir, H. \& Strömqvist, S. (2004). Discourse stance:Written and spoken language. Written Language and Literacy, 5(2), 255-289.

Berman, R. \& Nir-Sagiv, B. (2007). Comparing narrative and expository text construction across adolescence: A developmental paradox. Discourse Processes, 43(2), 79-I 20.

Berman, R. \& Ravid, D. (2009). Becoming a literate language user. Oral and written text construction across adolescence. En D. Olson \& N. Torrance (Eds.), The Cambridge Handbook of Literacy (pp. 92-I I I). Cambridge: Cambridge University Press.

Bruner, J. (1986). Actual minds, possible worlds. Cambridge, MA: Harvard University Press.

Fraurud, K. (1990). Definiteness and the processing of noun phrases in natural discourse. Journal of Semantics 7, 395-433.

Gundel, J., Herberg, M. \& Zacharski, R. (1993). Cognitive status and the form of referring expressions in discourse. Language, 69, 274-307.

Haviland, S. \& Clark, H. (1974). What's new? Acquiring new information as a process in comprehension. Journal of Verbal Learning and Verbal Behavior, I3, 51 2-21.

Hickmann, M. (1995). Discourse organization and the development of reference to person, space and time. En P. Fletcher \& B. MacWhinney (Eds.), The Handbook of Child Language (pp. 4|3-447). Oxford: Blackwell.

Hickmann, M. (2003). Children's discourse. Person, space and time across language. Cambridge: Cambridge University Press.

Karmiloff, K. \& Karmiloff-Smith,A. (2005). Hacia el lenguaje. Desde el feto al adolescente. Madrid: Morata.

Karmiloff-Smith, A. (1985). Language and cognitive processes from a developmental perspective. Language and Cognitive Processes, I, 6 I-85. 
Labov, W. (1972). Language in the inner city: Studies in the Black English Vernacular. Philadelphia: University of Pennsylvania Press.

Labov, W. \& Waletzky, J. (1967). Narrative analysis: Oral versions on personal experience. En J. Helm (Ed.), Essays on the verbal and visual arts (pp. 12-44). Seattle: University of Washington Press.

McCabe,A. \& Peterson, C. (I99I). (Eds.). Developing narrative structure. Hillsdale, NJ: Erlbaum.

Löbner, S. (1985). Definites. Journal of Semantics, 4, 279-326.

MacWhinney, B. (2000). The CHILDES project:Tools for analyzing talk. Mahwah, NJ: Erlbaum.

Ninio, A. \& Snow, C. (1996). Pragmatic development. Boulder, CO:Westview Press.

Nippold, M. (1998). Later language development. The school-age and adolescent years. Austin, TX: Pro ed.

Paprotté,W. \& Sinha, C. (1987).A functional perspective on early language development. En M. Hickmann (Ed.), Social and functional approaches to language and thought (pp. I86-202). Orlando:Academic Press.

Ravid, D. \& Berman, R. (2006). Information density in the development of spoken and written narratives in English and Hebrew. Discourse Processes, 4 I (2), I I7-I49.

Ravid, D. \& Tolchinsky, L. (2002). Developing linguistic literacy:A comprehensive model. Journal of Child Language, 29, 4I 7-447.

Recasens, M., Martí, M. \& Taulé, M. (2009). First-mention definites: More than exceptional cases. En S. Featherston \& S. Winkler (Eds.), The Fruits of Empirical Linguistics (pp. 768-793). Berlin: de Gruyter.

Slobin, D. (1990). Learning to think for speaking: Native language, cognition and rhetorical style. En A. Bocaz (Ed.), Actas del Primer Simposio sobre Cognición, Lenguaje y Cultura: Diálogo Transdisciplinario en Ciencias Cognitivas (pp. 129-152). Santiago: Universidad de Chile.

Slobin, D. (1996).Aspectos esenciales en la adquisición del español: Contribuciones a la teoría. En M. Pérez Pereira (Ed.), Estudios sobre la adquisición del castellano, catalán, eusquera y gallego (pp. 27-5I). Santiago de Compostela: Universidad de Santiago de Compostela.

Tolchinsky, L. (2007). El desarrollo de la narración oral y escrita más allá de la infancia. Coloquio Internacional: Las narrativas y su impacto en el desarrollo infantil. México DF: México.

Tolchinsky, L., Rosado, E., Aparici, M. \& Perera, J. (2005). Becoming proficient educated users of language. En D. Ravid \& H. Bat-Zeev (Eds.), Perspectives on Language and Language Development. Essays in Honor of Ruth Berman (pp. 375-389). Dordrecht/Boston/London: Kluwer.

Tomasello, M. (1998). The new Psychology of Language: Cognitive and functional approaches to language structure. Mahwah, NJ: Erlbaum.

Tomasello, M. (2005). Beyond formalities:The case of language acquisition. The Linguistic Review, 22(2-4), $183-197$.

Vion, M. \& Colas, A. (1999). Maintaining and reintroducing referents in French: Cognitive constraints and development of narrative skills. Journal of Experimental Child Psychology, $72,32-50$. 


\section{NOTAS}

I Se trata de un proyecto financiado por la Fundación Spencer de Chicago (Major Grant, Ruth Berman PI 1997-200 I, Universidad de Tel Aviv). Participaron como investigadores responsables de cada una de las lenguas involucradas: Judy S. Reilly, de San Diego State University; Harriet Jisa, Université Lyon 2, Hrafnhildur Ragnarsdóttir, Iceland University of Education, Sven Strömqvist, Lund University, Liliana Tolchinsky, Universitat de Barcelona y Janet van Hell y Ludo Verhoeven, University of Nijmegen.
${ }^{2}$ Sistema de formato estandarizado para transcribir muestras de interacciones conversacionales, cuyo nombre proviene de la sigla en inglés de Codes for the Human Analysis of Transcripts.

${ }^{3}$ La ortografía original de los textos no fue corregida en los ejemplos. Sí se ha modificado la segmentación original del corpus en cláusulas. En este caso las cláusulas se han agrupado por componente de estructura narrativa para facilitar la comprensión de los ejemplos. 